\title{
Proposing a Knowledge-based Model for Photovoltaic Modules Operating under Shading Conditions
}

\author{
FARHAD KHOSROJERDI ${ }^{1}$, STEPHANE GAGNON ${ }^{2}$, RAUL VALVERDE ${ }^{3}$ \\ ${ }^{1,2}$ Département d'informatique et d'ingénierie, \\ Université du Québec en Outaouais (UQO) \\ 283 Blvd. Alexandre-Taché, Gatineau \\ ${ }^{3}$ John Molson School of Business \\ Concordia University \\ 1455 Blvd. Maisonneuve O, Montréal \\ CANADA
}

\begin{abstract}
In a photovoltaic (PV) control system, the application of a maximum power point tracking (MPPT) method is the key factor that enables PV modules to operate efficiently under shading conditions. However, dealing with technical parameters of the MPPT-based controller requires expertise's knowledge about MPPT methods. PV planning tools overlook to provide system design parameters needed for the control system. Ontologies, as knowledge-based models, provide improved representation, sharing and re-use of the relevant information that facilitate the process of decision-making. In this work, we propose a knowledge-based model representing key concepts associated with an MPPT-based control device. The ontology model is featured with Semantic Web Rule Language (SWRL) allowing the system planner to extract information about MPPT classifications and select an appropriate MPPT method. Moreover, technical recommendations and design information needed for the control system is delivered as well. Using the proposed ontology helps nontechnical practitioners and end-users to define design-related parameters correctly and plan efficient PV systems.
\end{abstract}

Keywords: - MPPT, ontology, PV efficiency, knowledge-base model, MPPR-based controller

Received: August 31, 2020. Revised: November 11, 2020. Accepted: December 10, 2020. Published: December 29, 2020.

\section{Introduction}

A photovoltaic (PV) system as an environmentalfriendly and sustainable source of energy requires little maintenance $[1,2]$. Since 25 years ago, it has become one of the main contributors among other forms of renewable energy resources [3]. Solar energy productions have been grown more than 70,000 MW [4]. Therefore, its significant role in the future of energy resources cannot be denied. The convenience of PV system installations has motivated residential and commercial consumers to consider it as an important source of energy. In Canada, the domestic market has been growing on average at about $26 \%$ per year since 1993 and about $48 \%$ since 2000 [5]. As a result, consumers with minimum or basic knowledge about the components of a PV system attempt to install such systems. However, determining components of an efficient system requires an expert's knowledge, especially when it operates under shading conditions [6]. Shadings are caused by nearby objects or environmental elements including buildings, trees, clouds, dust, snow, and pollution. The accumulation of various particles, climate or ambient related, will result in generating less power.

To overcome the low efficiency of a PV system operating under shading, maximum power point tracking (MPPT) methods are used, allowing the PV system to perform in its optimal operation. An MPPT-based control system implements the algorithm that controls the generated power. The application of an MPPT method and defining certain parameters associated with the control system noticeably improve efficiency of the PV system. However, technical requirements of the application would be quite complex for non-technical installers. The complexity includes choosing an appropriate algorithm, determining its parameters, initial values, and further, important components of the control system. Non-technical consumers employ software tools for planning PV systems. They rely on power estimation reports provided by these planning tools. Nevertheless, even the most reliable software, System Advisor Model (SAM), overlooks the 
decline of power generations caused by snowfalls. Yet, there is no PV planning tool that offers information or any type of data about MPPT methods.

In this work, we propose a knowledge base ontology model that represents key concepts associated with the designing of an MPPT-based controller. The proposed ontology aids to determine technical constraints and requirements of the control system and to select an appropriate MPPT method. In addition, the ontology model presents system design recommendations and suggestions that most PV planning tools fail to report. The designed ontology, named MPPT-On, is developed using SWRL rules and queries. Evaluation of the proposed ontology is performed using a case study. The SAM simulation is used for planning a PV project. Then, we employ the proposed model to extract information needed to deal with MPPT controllers. The defined rules and queries provide valuable technical recommendations and design considerations that help system designers and project managers in different phases of a PV project.

This paper is structured as follows: the next section discusses problems in designing an efficient PV system. In section 3, the processes of designing and developing the proposed ontology is described. The ontology evaluation is also stated in this section. Finally, the conclusion is presented in section 4 .

\section{Problems in Designing an Efficient PV System}

PV systems can be designed stand-alone or gridconnected depending on the application. Standalone PV systems normally deliver power to a single load or off-grid network of electric loads. Gridconnected PV systems deliver power to the grid and can interact with the power network [7]. The overall topology of a PV system is shown (see Fig. 1). An MPPT algorithm modulates the duty cycle for the converter and enables PV system to perform in its maximum efficiency. A PV system is built in a series-parallel configuration to produce desire output power and voltage. In practical, they are connected in series to form a module of 36,60 , or 70 cells. Then, the modules are assembled in different series and parallel configurations to form an array at the desired output voltage and current [8$11]$.

\subsection{MPPT Methods: A Literature Review}

Classifications of existing methods representing functionality of the algorithms are widely distinctive. These perceptions mainly focuses on MPPT applications, optimization methods, costs, parameters used, efficiencies, tuning parameters, the system complexity, and the rapid convergence [1216]. Ultimately, the most common clustering can be defined as: I) conventional or classical methods, II) modern or soft computing methods, and III) hybrid methods, and IV) power electronics (PE) based methods. Conventional methods offer convenience and simplicity [17]. However, they may be trapped in local points and detect one of the local points as the MPP for the system consisting of several PVs performing under PSCs.

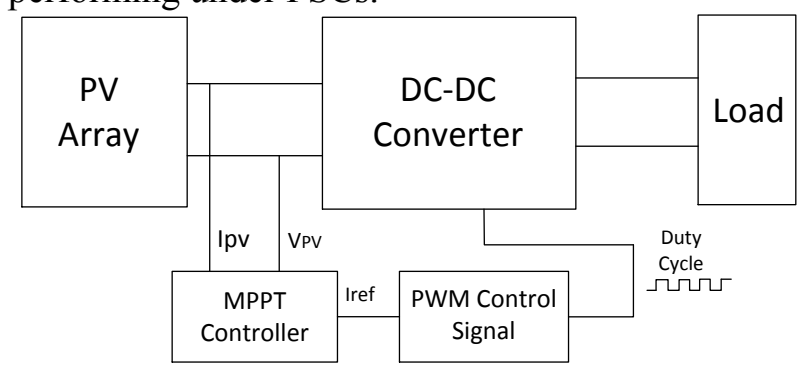

Fig. 1. A typical and simple PV system

Furthermore, they provide lower efficiency and convergence speed comparing to soft computing methods $[13,15]$. Still, they play important roles in engineering applications based on their simplicity, flexibility, gradient-free mechanism, and capability of searching global optima $[18,19]$. Soft computing methods can be categorized into the artificial intelligence (AI) and meta-heuristic optimization techniques [18, 20, 21]. Meta-heuristic methods can be categorized into two subdivisions, the evolutionary algorithm (EA) and population-based or swarm intelligence (SI) methods. SI techniques mimicking evolution and social behaviour of creatures in nature [19]. Some researchers have been improved conventional and soft-computing approaches by hybridizing these techniques. It means that some studies have modified one method or combine two methods from different MPPT classifications and improve the functionality of the origin algorithm [22]. Therefore, combination of any method in each category with another approach can result in developing a hybrid method. Obviously, due to the complexity of their algorithms, applications of these methods in realworld are questionable. In fact, implementation of an MPPT algorithm is an important technical concern leading us to add a new branch to the MPPT classification. Employing hardware and 
technical features of the power electronics components are the reason they are called PE-based methods. In a previous work [23], we studied these methods and highlighted the importance role of microcontroller-based (MCU-based) MPPT techniques. Unlike numerous studies concentrating on developing redundant soft computing MPPT algorithms, major elements of a PV system and its architecture are briefly described as important factors to improve performance of PV systems under PSCs. It is notified that advanced features of nowadays' MCUs such as temperature and irradiance sensors as well as Wi-Fi connectivity can be developed in the context of power conversion. As a result, we add PE-based methods to the usual MPPT methods classification.

\subsubsection{Challenges in the Efficiency Assessment}

In a previous published paper [24], efficiencies of $\mathrm{P} \& \mathrm{O}$ method are assessed when the simulation model experiencing three different input patterns, respecting irradiance. It has been shown that substantial alterations in an irradiance pattern marks in lower efficiency with a greater number for the iteration number. The results of the work indicate that the simulation must be implemented by experiencing various irradiance shapes. In addition, applying unrealistic irradiance patterns to the Simulink model establish unrealistic efficiency results. In this regard, the reliability and accuracy of simulations presenting poor efficiency for the $\mathrm{P} \& \mathrm{O}$ method can be challenged. The fact that there are no standard irradiance patterns to evaluate accuracy of a simulation, the accuracy and reliability of the MPPT methods and their efficiencies are questionable. Testing multiple conditions and considering local irradiance data can assist system designers to pick an optimum MPPT method. The simulated irradiance patterns and their volatility should reflect real shading conditions based on the local climate data.

As a result, it is argued that the efficiency percentage or the convergence speed related to any method can be altered when certain adjustments performed. Even conventional methods may perform a better efficiency than an EA-based technique. It means that parameters of a PV installation can be adjusted based on several variables depending on the microcontroller, control parameters chosen ( $I$ or $V$ ), and configurations of PV arrays. Therefore, it reveals that choosing an MPPT algorithm from existence classifications does not guarantee a certain efficiency percentage, because of: 1) adjustments applied in experiments related to the classifications, 2) initial values defined in EA-based and SI-based algorithms, and 3) various PV system architectures.

\subsection{PV Planning Tools}

A PV system planning tool (sometimes referred to and known as a designing tool as well) estimates the energy production and cost of energy of gridconnected PV energy systems. The output reports and results of the PV design tool require a reliable PV performance model representing the real-world behaviours of the system. There are online applications and software products freely available for PV system planning. They allow project managers, utility consumers, technology developers, and researchers to easily predict the electricity output of a PV system and evaluates the system performance. These planning tools provide performance predictions and cost of energy for gridconnected PV projects based on installation and operating costs and system design parameters selected by an end-user from databases and data libraries. These tools provide decision-making support systems through which PV planning can be achieved efficiently.

For the case of submitting a reliable PV planning software to be used as a case study later, we investigated 31 commercial and open source PV planning/designing software tools using Google search. The complete list is available in [25]. These planning tools provide PV system planning or designing. The level of accuracy and availability of technical information were reviewed in the Help pages, technical references, manuals, software presentations, demo videos, and commercial emails. The followings present criteria and overall considerations for the selection: is a PV design or planning application included on the website? is the trial version run and executed completely? how reliable and accurate were related databases and meteorological data? what type of PV model is used in the simulation? what type of technical and scientific information are presented?

The final list of the most reliable PV planning tools included: PVWatts, SAM, HOMER Pro, RETScreen Expert, PVsys, Pollysun, SolarPro, PVSOL, and PV Perform Mod. The National Renewable Energy Laboratory (NREL), the national laboratory of the U.S. Department of Energy, and the National Research Council (NRC), and the government of Canada's largest research organization, offer free of charge PV designing services. The NREL's model employs an accurate PV performance model, capable of detecting PSCs and other related DC losses. The developed performance model, SAM, calculates the hourly 
output of a renewable energy system over a single year. Therefore, we select the SAM model as the most reliable and accurate simulation used for planning PV systems. The ontology evaluation is performed against this model to verify the benefits of the proposed ontology.

\section{The Proposed Ontology}

An ontology is "an explicit specification of a conceptualization" that can be interpreted as formally describing a domain of interest through an abstract model [26]. In this way, the community of a certain domain can reuse and develop the shared knowledge constructed with alike terminology. Ontologies are agreements about share conceptualizations including conceptual frameworks for modeling knowledge and representation of specific domain knowledge [27]. They are known as specific frameworks of representational vocabulary and common terminology. They provide hierarchy form of specified classes and their relationships. Interconnection across heterogeneous applications conveniently provides an understandable framework for developing ontologies and facilitate them with up-to-dated knowledge and elaborated semantics [28]. Ontology can deal with large volumes of data, share knowledge, and incorporate the relevant domain concepts as well as their associated relations [29].

\subsection{The Ontology Design}

In this step, the scope of the ontology and its domain are developed. We identify semantics and different concepts related to MPPT methods in the PV domain. There are several ontology methodologies for developing an ontology including Methontology [30], On-To-Knowledge [31], NeOn [32], and Horrocks Ontology Development Method [33]. While these methodologies have been utilized in several knowledge base domains, we need to apply a method that offers convenience technologies working with many software environments. Ontology Development 101 is the well-known and most practiced methodology for developing ontologies [34]. The concept of Ontology Development 101 is adopted for developing the proposed ontology. In the methodology, four main activities need to be defined [34]: 1) different terms in the domain and relations among them, 2) concepts (classes) in the domain, 3) hierarchy arrangement of the concepts (sub-classes and classes relationships), 4) constraints, values, and properties values.
This methodology presents technologies to build an ontology from starting point. It applies OWL language and conveniently implemented in ProtégéOWL editor that includes several compatible plugins for different applications. As defined in [35], "Protégé is a free, open-source platform that provides a growing user community with a suite of tools to construct domain models and knowledgebased applications with ontologies." It is an ontology development environment that allows to create, upload, modify, and share ontologies. It supports OWL 2 Web Ontology Language and description logic reasoners like Hermit and Pellet [35]. There are key concepts used in Protégé including individuals, classes, and properties. Individuals are known also as instances can be referred to as being "instances of classes." Classes contain all the individuals that are categorized in a domain of interest. Classes may be organized into a superclass or sub-class hierarchy, which is also known as a taxonomy. A class represents a concept in the domain or a collection of elements with similar properties. Properties are binary relations on individuals connecting two individuals together. Properties describe attributes of instances of the class and relations to other instances. Object properties are relationships between two individuals. Data properties describe relationships between individuals and data values. Annotation properties can be used to add information (metadata-data about data) to classes, individuals, and object/data properties.

We implement the following steps to construct our ontology:

1) creating the class hierarchy

2) defining the OWL properties: defining their type (functional, transitive, symmetric, reflexive, etc.), and defining their domain/range as per need

3 ) describing and defining the classes created for example restrictions (axioms)

4) invoking reasoner, checking the consistency of the ontology, and creating the inferred view

5) Creating certain individuals by assigning certain OWL properties

6) Running the reasoner and check it.

Fig. 2 depicts a view of the object properties and data properties defined for the ontology in Protégé. Fig. 3 illustrates the graphical representation of the proposed model including super-classes and their relationships. 


\subsection{SWRL Rules and Queries for MPPT Methods}

SWRL rules are designed to provide information about the technical and physical characteristics of the MPPT-Based controller. These information are essential for the PV system designed by a PV planning software. During the processes of identifying class axioms, three areas were detected as the main resource of concepts in the context of MPPT including the methods, characteristics, and control system.

MPPT methods represent knowledge base about the algorithms, different techniques, parameters involved, mathematical approaches employed, and related variables. Searching techniques used for tracking GP and functions as well as their parameters are the intentions of any research endeavors about MPPT methods. Unlike these notions, MPPT-linked semantics without physical or technical features are classified in the sector of MPPT characteristics. These classes represent criteria and measures that an MPPT approach can be compared with the another. The third key knowledge area in the context of the MPPT domain belongs to the hardware of an MPPT-based control system.

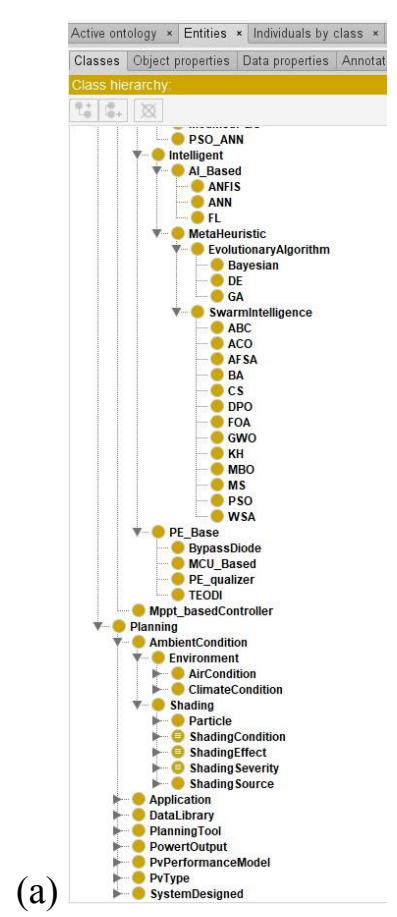

(b)

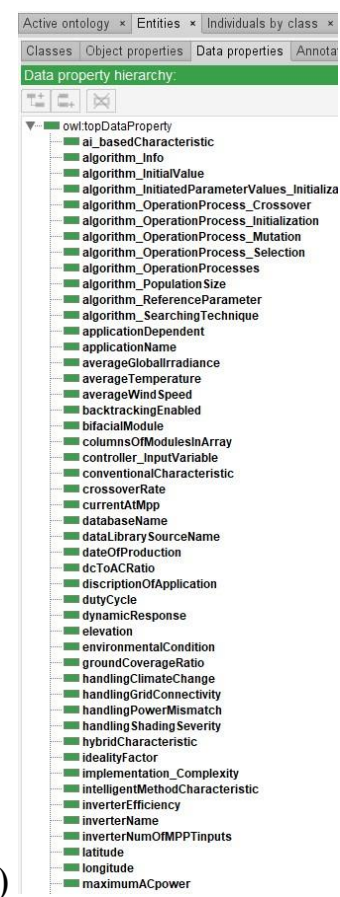

(c)

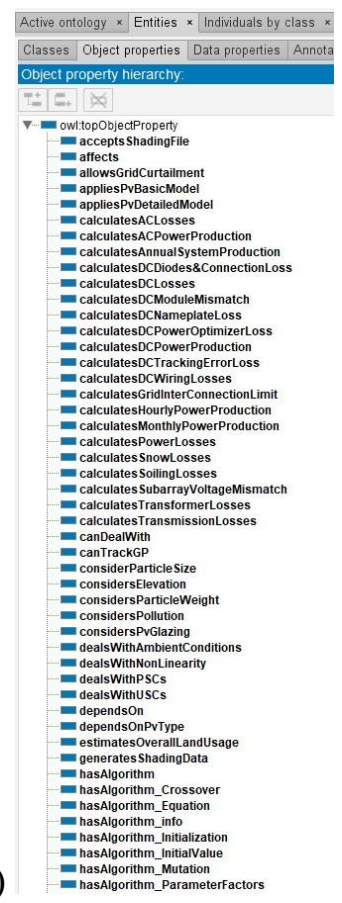

Fig. 2 Snapshot views of the proposed ontology built in Protégé: (a) Class hierarchy, (b) data properties, (c) object properties 


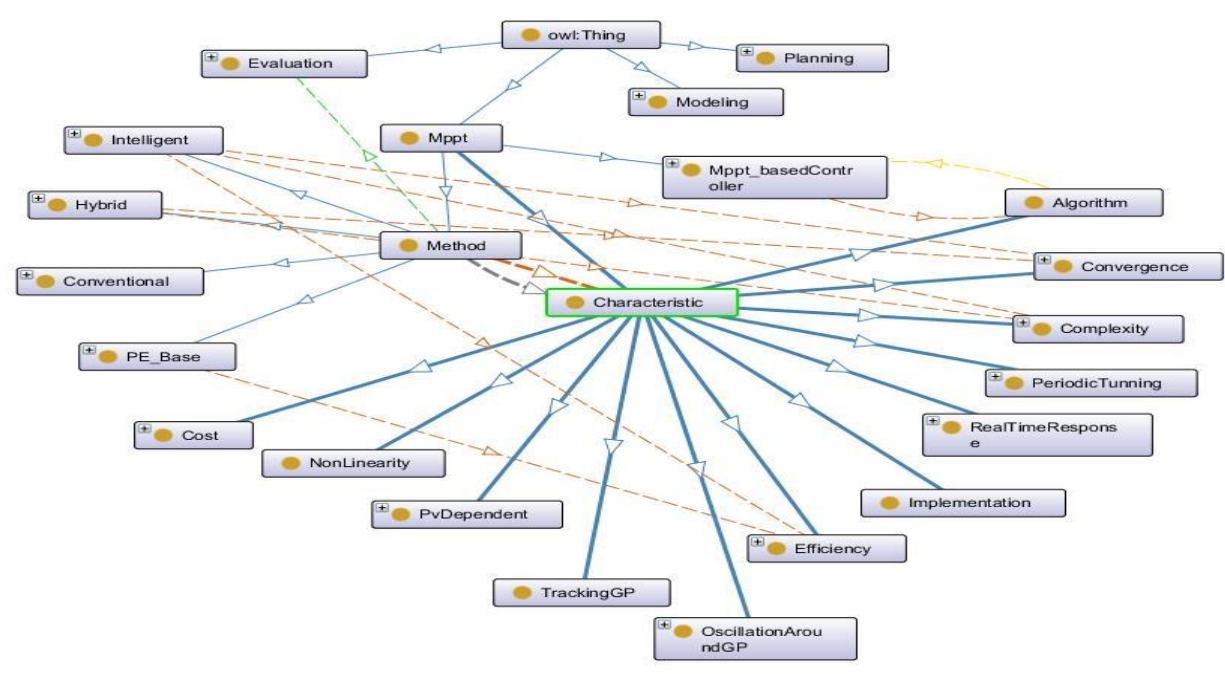

Fig. 3 A graphical representation for MPPT characteristics and its classifications

Technical features and physical properties of the controller are embodied in this stream. These properties are defined in the ontology so that the important properties of an MPPT method can be obtained. Fig. 4 outlines these data properties from which the SWRL rules are extracted. The prioritized numbers shown in the figure states how important is the SWRL rules. In other words, defining rules for MPPT algorithms alone can be useful for the MPPT database.
PV planners can present the information of the method algorithm to the system designer for technical purposes. The defined SWRL rules can produce different reports associated with MPPT methods. The aid of the model is to provide technical recommendations and design information needed for an MPPT controller. SQWRL queries are employed to define various rules and extract information from the MPPT database added to the ontology as instances.

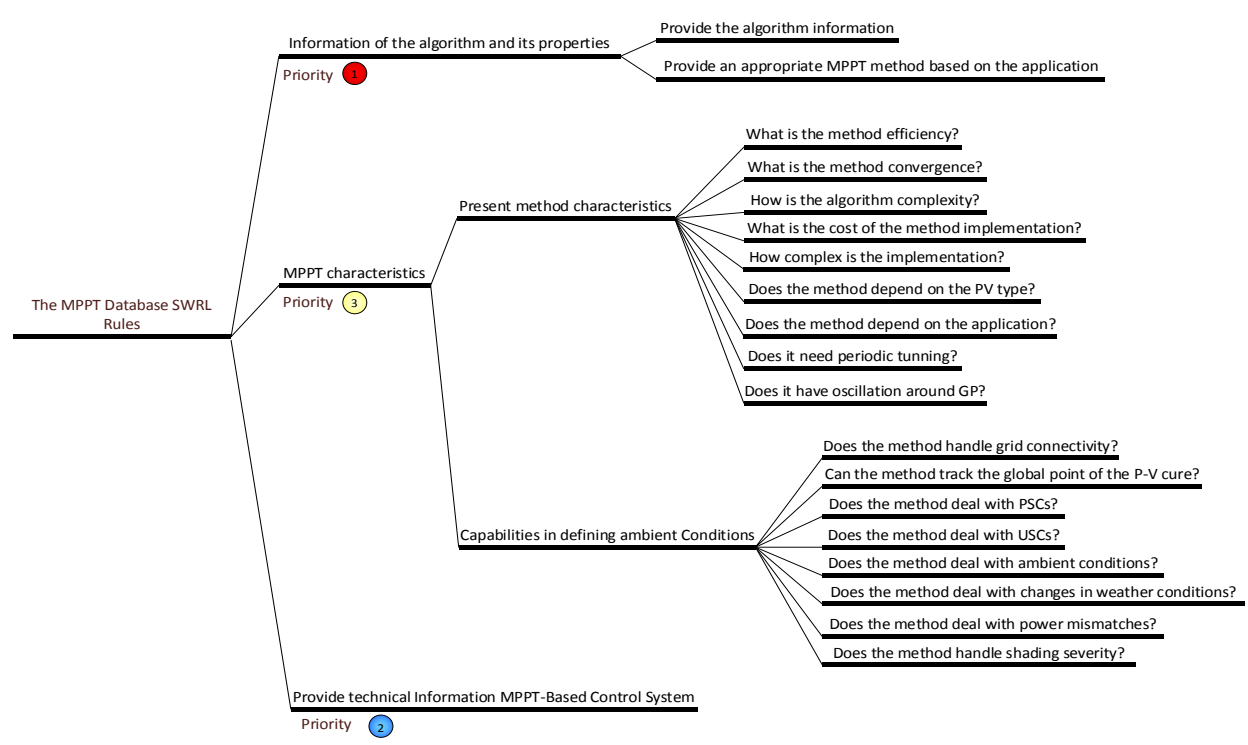

Fig. 4 The structure of SWRL rules for the proposed MPPT database 
SWRL rules, in fact, act to evoke knowledge out of the ontology model instead of manipulating data or changing values of a class assertion and produce new class assertions.

\subsection{Evaluation of the Proposed Model}

The evaluation of an ontology is as important as developing it. Evaluation can be deemed as an approval for the application of a developed ontology. It indicates that how suitable the ontology model is for which it is supposed to used. The proposed ontology is semantically validated by a case study.

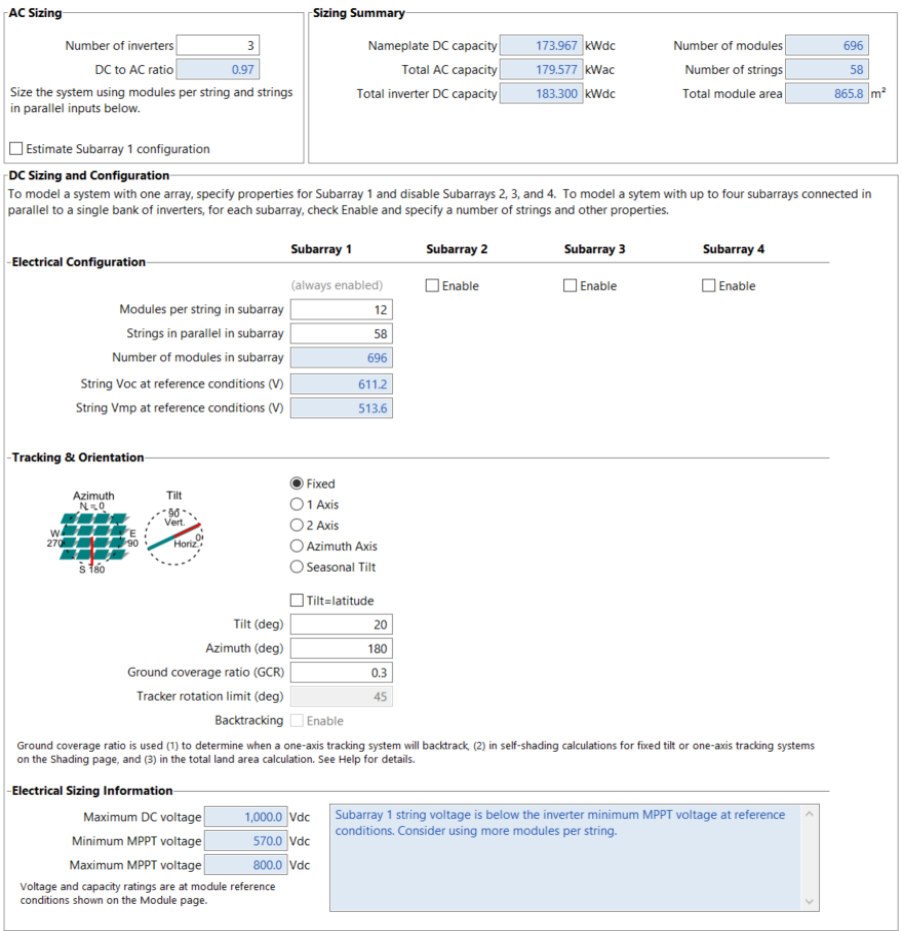

Fig. 5 Technical characteristics and sizing summary of the case study

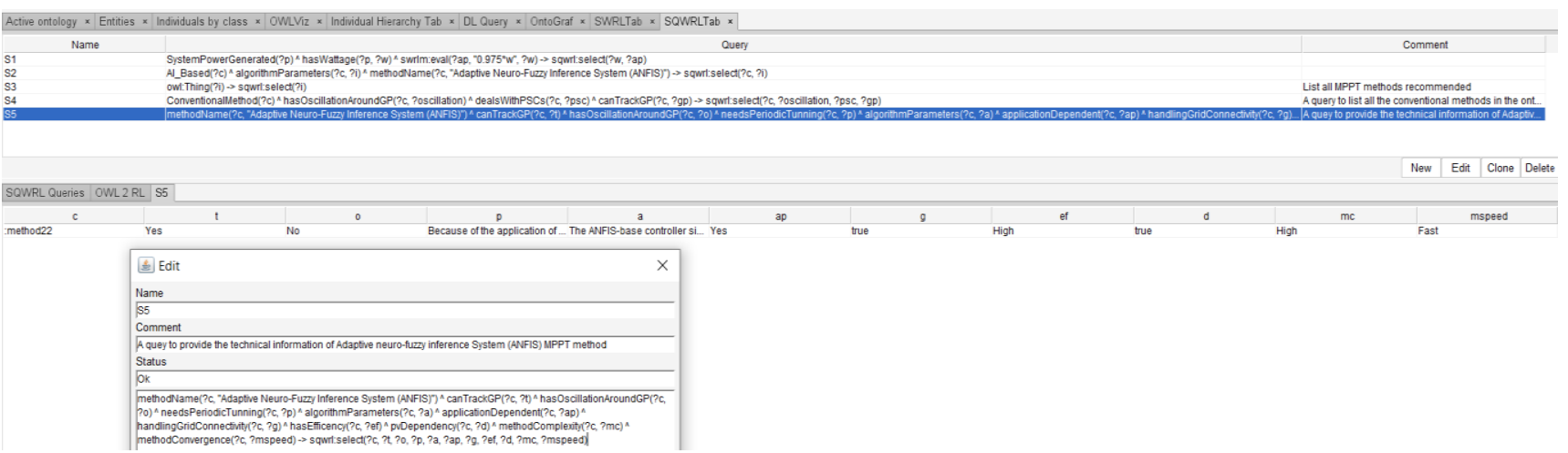

Fig. 6 SWRL query and its result: information about the ANFIS method

\subsection{The Application of Proposed Ontology}

The methods are added to the ontology as instances building an MPPT database that offerings technical information needed for designing the
Using the SAM simulation, we design a PV power generation system capable of producing 179.77 $(\mathrm{kW}) \mathrm{AC}$ power located in Montreal, QC, Canada. The technical characteristics and the sizing summary of the system designed is presented in Fig. 5. The SAM model provides several reports presenting hourly and monthly power productions, while there are no technical specifications about the MPPT-based control system. Therefore, the designed system represents no information about the MPPT-based controller. 
hardware. For the case study, we decide to use an AI method for the controller. Thus, the instances are reviewed, and the associate rule is implemented to extract information of an AI-based method. The query to extract the technical information of Adaptive neuro-fuzzy inference System (ANFIS) MPPT method is chosen due to its significant performances coping with shading conditions.

The rule "Method22" of the ontology introduces data related to the ANFIS method, which is an AI technique. Fig. 6 depicts the SWRL rule of its result presenting information related to the ANFIS method. It indicates that the method can track GP, depends on PV type, provide fast convergence with high efficiency. However, the application of its algorithm is difficult to implement. The proposed ontology and SWRL rules and queries are available in [25].

\section{Conclusion}

In this work, we have proposed an ontology model representing MPPT methods and characteristics. The defined ontology reasoning assists end-users to plan efficient PV systems under various ambient conditions. The proposed model has been validated using a real-world PV project as a case study.

The model consists of all essential parameters and factors required for designing MPPT-based control systems. These parameters are presented in the forms of OWL class axioms, and their characteristics are defined as objective properties and data properties. Technical attributes are specified in the ontology allowing PV planners and practitioners to determine several elements of $\mathrm{PV}$ design. Moreover, valuable planning and designing recommendations in the form of queries can be extracted by exploring the rules.

The only factor that could be perceived as a setback for using the proposed ontology is that the user must be familiar with the environment of Protégé, running the SWRL rule and query. In other words, the ontology model is not performing any function by its own and requires a user to apply it. This limitation can be eliminated in the future studies by developing an application to implement the ontology when the end-suer use it.
References

[1] A. M. S. Aldobhani and R. John, "Maximum power point tracking of PV System using ANFIS prediction and fuzzy logic tracking," presented at the The International Multiconference of Engineers and Computer Scientists Hong Kong, 19-21 March, 2008, 2008.

[2] B. Parida, S. Iniyan, and R. Goic, "A review of solar photovoltaic technologies," Renewable and Sustainable Energy Reviews, vol. 15, no. 3, pp. 1625-1636, 2011.

[3] A. Bloom et al., "It's Indisputable: Five Facts About Planning and Operating Modern Power Systems," IEEE Power and Energy Magazine, vol. 15, no. 6, pp. 22-30, 2017.

[4] M. Seyedmahmoudian et al., "State of the art artificial intelligence-based MPPT techniques for mitigating partial shading effects on PV systems - A review," Renewable and Sustainable Energy Reviews, vol. 64, pp. 435-455, 2016.

[5] Y. Poissant and A. C. Vikis, "Overview of photovoltaic solar cell R\&D capability in Canada ED. 4," in "CanmetENERGY, Varennes Research Centre," Natural Resources Canada2013.

[6] S. Subiyanto, A. Mohamed, and M. A. Hannan, "Intelligent maximum power point tracking for PV system using Hopfield neural network optimized fuzzy logic controller," Energy and Buildings, vol. 51, pp. 29-38, 2012.

[7] W. Na, T. Carley, L. Ketcham, B. Zimmer, and P. Chen, "Simple DSP Implementation of Maximum Power Pointer Tracking and Inverter Control for Solar Energy Applications," Journal of Power and Energy Engineering, vol. 04, no. 09, pp. 6176, 2016.

[8] F. Salem and M. A. Awadallah, "Detection and assessment of partial shading in photovoltaic arrays," Journal of Electrical Systems and Information Technology, vol. 3, no. 1, pp. 23-32, 2016.

[9] B. A. Alsayid, S. Y. Alsadi, J. f. S. Jallad, and M. H. Dradi, "Partial Shading of PV System Simulation with Experimental Results," Smart Grid and Renewable Energy, vol. 04, no. 06, pp. 429-435, 2013. 
[10] M. Seyedmahmoudian et al., "Simulation and Hardware Implementation of New Maximum Power Point Tracking Technique for Partially Shaded PV System Using Hybrid DEPSO Method," IEEE Transactions on Sustainable Energy, vol. 6, no. 3, pp. 850-862, 2015.

[11] R.-1. Tang, Z. Wu, and Y.-j. Fang, "Maximum power point tracking of largescale photovoltaic array," Solar Energy, vol. 134, pp. 503-514, 2016.

[12] B. Subudhi and R. Pradhan, "A comparative study on maximum power point tracking techniques for photovoltaic power systems," IEEE Trans. Sustain. Energy, pp. 89-99, 2013.

[13] A. Gupta, Y. K. Chauhan, and R. K. Pachauri, "A comparative investigation of maximum power point tracking methods for solar PV system," Solar Energy, vol. 136, pp. 236-253, 2016.

[14] P. Joshi and S. Arora, "Maximum power point tracking methodologies for solar PV systems - A review," Renewable and Sustainable Energy Reviews, vol. 70, pp. 1154-1177, 2017.

[15] S. Lyden and M. E. Haque, "Maximum Power Point Tracking techniques for photovoltaic systems: A comprehensive review and comparative analysis," Renewable and Sustainable Energy Reviews, vol. 52, pp. 1504-1518, 2015.

[16] A. R. Jordehi, "Maximum power point tracking in photovoltaic (PV) systems: A review of different approaches," Renewable and Sustainable Energy Reviews, vol. 65, pp. 1127-1138, 2016.

[17] M. A. Elgendy, B. Zahawi, and D. J. Atkinson, "Assessment of the incremental conductance maximum power point tracking algorithm," IEEE Transactions on Sustainable Energy, vol. 4, p. 9, 2013.

[18] S. Mirjalili, S. M. Mirjalili, and A. Lewis, "Grey Wolf Optimizer," Advances in Engineering Software, vol. 69, pp. 46-61, 2014.

[19] S. Mirjalili, S. Saremi, S. M. Mirjalili, and L. d. S. Coelho, "Multi-objective grey wolf optimizer: A novel algorithm for multicriterion optimization," Expert Systems with Applications, vol. 47, pp. 106-119, 2016.

[20] Z. Salam, J. Ahmed, and B. S. Merugu, "The application of soft computing methods for MPPT of PV system: A technological and status review," Applied Energy, vol. 107, pp. 135-148, 2013.

[21] A. Reza Reisi, M. Hassan Moradi, and S. Jamasb, "Classification and comparison of maximum power point tracking techniques for photovoltaic system: A review," Renewable and Sustainable Energy Reviews, vol. 19, pp. 433-443, 2013.

[22] S. Saravanan and N. Ramesh Babu, "Maximum power point tracking algorithms for photovoltaic system - A review," Renewable and Sustainable Energy Reviews, vol. 57, pp. 192-204, 2016.

[23] F. Khosrojerdi and N. H. Golhkandan, "Microcontroller-based Maximum Power Point Tracking Methods in Photovoltaic systems," in 9th Power Electronic and Drive Systems and Technologies Conference (PEDSTC), Tehran, Iran, 2018: IEEE.

[24] F. Khosrojerdi and N. Mazlum, "Effects of Irradiance Patterns on Efficiency of a Photovoltaic System Controlled by the Perturbation and Observation Method," in 9th PEDSTC, Tehran, Iran, 2018, p. 6: IEEE.

[25] F. Khosrojerdi. (2020). The Proposed Ontology (MPPT-On) and Associateted Files. Available: https://github.com/khof01/ontology

[26] T. R. Gruber, "A Translation Approach to Portable Ontology Specifications," in "Knowledge Systems Laboratory," Computer Science Department Stanford University, Knowledge Acquisition1992, vol. 5.

[27] T. R. Gruber, "A translation approach to portable ontologies," Knowledge Acquisition, vol. 92, no. 71, pp. 199-220, 1993.

[28] E. Kontopoulos, G. Martinopoulos, D. Lazarou, and N. Bassiliades, "An ontologybased decision support tool for optimizing domestic solar hot water system selection," Journal of Cleaner Production, vol. 112, pp. 4636-4646, 2016.

[29] M. Rospocher, C. Ghidini, and L. Serafini, "An ontology for the Business Process Modelling Notation," Artif. Intell., 2014.

[30] M. Ferndndez, A. Gomez-Perez, and N. Juristo, "Methontology: From Ontological Art Towards Ontological Engineering," 1997. 
[31] D. Fensel, F. V. Harmelen, M. Klein, and H. Akkermans, "On-To-Knowledge: Ontologybased Tools for Knowledge Management," p. 7, 2000 .

[32] M. C. Suárez-Figueroa, A. Gómez-Pérez, and M. Fernández-López, "The NeOn Methodology for Ontology Engineering," in Ontology Engineering in a Networked World, 2012, pp. 9-34.

[33] K. Breitman, M. A. Casanova, and W. Truszkowski, Semantic Web Concepts, Technologies and Applications. SpringerVerlag Ltd., London, UK, 2007.

[34] N. F. Noy and D. L. McGuinness, "Ontology Development 101: A Guide to Creating Your First Ontology," Stanford University, Stanford, CA,2001.

[35] Protégé. (2020). Protégé Products. Available:

https://protege.stanford.edu/products.php

\section{Creative Commons Attribution License 4.0 (Attribution 4.0 International, CC BY 4.0)}

This article is published under the terms of the Creative Commons Attribution License 4.0

https://creativecommons.org/licenses/by/4.0/deed.en US 